\title{
RE-VISITANDO LA TEORÍA ESPACIAL DEL VOTO: \\ NUEVA EVIDENCIA SOBRE CHILE AL 2003 Y SUS \\ IMPLICANCIAS PARA LAS CANDIDATURAS \\ PRESIDENCIALES DEL 2005
}

\author{
Claudio A. Bonilla \\ y Ernesto Silva M.
}

\begin{abstract}
Existen pocos estudios empíricos que apliquen modelos de economía política al contexto latinoamericano. El presente artículo utiliza la metodología Cahoon-Hinich para estimar modelos espaciales del espectro político chileno al año 2003. Los resultados, señalan los autores, reafirman la tesis de Mainwaring y Torcal (2003) en el sentido de que la sociedad chilena aún se encuentra polarizada, como lo estaba al terminar el gobierno militar (1973-1990) en el eje izquierda-derecha. Al incorporar al análisis el eje reforma versus statu quo, surgen interesantes resultados para entender el posicionamiento de quienes se preparaban para ser candidatos presidenciales el año 2005.
\end{abstract}

Claudio A. Bonilla. Ph.D. y Master en Economía de la Universidad de Texas en Austin, especialista en economía política y finanzas. Investigador de la Facultad de Economía y Negocios de la Universidad del Desarrollo. (cbonilla@udd.cl)

ERnesto Silva. Magíster en Políticas Públicas de la Universidad de Chicago y candidato a Doctor en Ciencia Política de la Universidad Autónoma de Madrid, especialista en políticas públicas y economía política. Investigador de la Facultad de Gobierno de la Universidad del Desarrollo (esm@udd.cl).

Estudios Públicos, 109 (verano 2008). 


\section{INTRODUCCIÓN}

\section{Black (1958) introdujo el famoso resultado del "votante mediano"} basado en una idea que Hotelling (1929) había pensado 30 años antes. Este resultado intuitivamente nos dice que para ganar una elección basta con convencer al votante “centrista”. Para que lo anterior sea cierto se requiere de ciertos supuestos bastante restrictivos (elección unidimensional ${ }^{1}$, preferencias con un solo peak, etc.). Sin embargo, la proposición que formuló Black hace cincuenta años permanece absolutamente vigente hasta el día de hoy en cualquier campaña electoral. Posteriormente Davis y Hinich (1966 y 1968), Romer y Rosenthal (1978), Cox (1990) y Hinich y Munger (1994), entre otros, expandieron el resultado del votante mediano a situaciones menos restrictivas que las inicialmente expuestas por Black. Con el tiempo el nivel de complejidad matemática asociada a este tipo de modelos ha crecido enormemente, en especial después del interés que el tema de las votaciones ha despertado en investigadores del área matemática que incursionan en las ciencias sociales. Lamentablemente, el desarrollo y la sofisticación de la teoría no han ido igualmente de la mano con el desarrollo de aplicaciones empíricas que testeen la teoría.

Sin embargo, a pesar de que existe una brecha entre el desarrollo teórico en economía política y sus aplicaciones empíricas, de todas formas se han desarrollado algunas metodologías para testear versiones más simples de estos modelos teóricos. Las dos principales metodologías aplicadas en la literatura son la de Poole (Poole y Rosenthal, 1984 y 1991), que utiliza datos en forma binaria y métodos no paramétricos para estimar las posiciones de los candidatos en una o dos dimensiones, y la metodología de Cahoon-Hinich (Cahoon, 1975, y Cahoon, Hinich y Ordeskook, 1978), que usa puntajes de termómetros ${ }^{2}$ para estimar las posiciones de los candidatos $\mathrm{y}$ los votantes en un espacio con dos dimensiones.

El objetivo de este estudio es aplicar la metodología de CahoonHinich (la cual será explicada más adelante) para construir mapas espacia-

1 "Unidimensional" se refiere a que un solo atributo del candidato es suficiente para que el votante elija. Por ejemplo, la posición del candidato en el eje izquierdaderecha.

${ }^{2}$ Los mapas espaciales corresponden a una gráfica en dos dimensiones de las posiciones de política pública que los votantes atribuyen a los distintos candidatos. La primera dimensión corresponde a la ubicación del candidato en el eje político-económico tradicional de izquierda-derecha. La segunda dimensión corresponde al eje reforma versus statu quo. 
$\operatorname{les}^{3}$ de las posiciones políticas de una serie de potenciales candidatos a la presidencia de Chile con datos a enero del 2003, y de esta forma ver si los resultados de Mainwaring y Torcal (2003) acerca de la polarización del espectro político chileno ${ }^{4}$ —basados en datos de 1995- son efectivamente válidos ocho años después. De igual forma, el estudio busca explicar el mapa político que permitió —un año después— el surgimiento de "candidatos presidenciales diferentes” en la Concertación, específicamente el caso de Soledad Alvear y Michelle Bachelet. Es importante hacer notar que a enero del 2003 Soledad Alvear ya era una posible candidata a la presidencia de la república, y por lo tanto está incluida en la muestra. Michelle Bachelet, por otro lado, no se perfilaba aún como una posible candidata, y es por esa razón que no está incluida en la muestra. Sin embargo, los resultados empíricos de nuestro análisis muestran claramente que el camino al éxito para la Concertación en las presidenciales del 2005 estaba por el lado de llevar "candidatas presidenciales diferentes".

El principal resultado de nuestro estudio es la constatación de un grado de polarización relativamente importante en el espectro político chileno a enero del 2003, lo cual muestra una persistencia en los resultados de Maiwaring y Torcal. Además, los mapas espaciales construidos con nuestros datos permiten entender el escenario político que enfrentaban las dos principales coaliciones chilenas al año 2003. De igual forma, los resultados de este trabajo permiten entender la irrupción de Michelle Bachelet como candidata presidencial, toda vez que en su condición de mujer y con énfasis diferentes a los candidatos tradicionales de la Concertación, logra posicionarse en un buen nivel de la dimensión reforma en la percepción de los votantes. Este planteamiento se hace extensivo también a la realidad de Soledad Alvear al año 2003, constituyendo una opción reformadora aun cuando haya sido ministra de distintas carteras durante buena parte del tiempo que su coalición llevaba en el poder.

\section{LITERATURA RELACIONADA}

Dow (1998) y González (2000) construyeron mapas espaciales para Chile usando una base de datos de un mes antes de la primera elección

${ }^{3}$ Un puntaje de termómetro mide qué tan cercano se siente un votante a un candidato. Por ejemplo, en una escala de uno a siete, si un votante piensa que un candidato no aplicará políticas públicas que sean beneficiosas para él, entonces le asignará un uno, por lo tanto decimos que ese votante se percibe frío con respecto al candidato. Por el contrario, si un votante cree que un candidato haría exactamente lo que es bueno para él y su familia, le asignará un siete.

${ }^{4}$ Más adelante en el trabajo describiremos las principales coaliciones, partidos y candidatos que existen en la política chilena. 
presidencial que tuvo lugar al término del gobierno militar en 1989. Como era de esperar, los resultados obtenidos en esos estudios muestran la profunda polarización que la sociedad chilena vivía al término del régimen. Bonilla (2002), usando una base de datos del año 2001 correspondiente a un solo distrito de la Región Metropolitana ${ }^{5}$, construyó mapas espaciales para ese distrito y encontró que los candidatos se distribuían de manera más o menos uniforme en el eje político económico izquierda-derecha, lo cual nos podría llevar a pensar que la potencial polarización postulada por Mainwaring y Torcal ya no estaría presente; sin embargo esos resultados están seriamente limitados por constituir una muestra distrital y no pueden ser extrapolados a resultados nacionales.

Aplicaciones empíricas de modelos de economía política a nivel internacional existen para Estados Unidos (Enelow y Hinich, 1984; Ghobarah, 2000), Taiwán (Lin et al., 1996), Alemania (Pappi y Eckstain, 1998), Ucrania (Hinich, Khmelko y Ordershook, 1999) y Turquía (Karkoglú y Hinich, 2006), entre otras.

\section{EL MODELO CLÁSICO EN ECONOMÍA POLÍTICA}

La economía política ha evolucionado en las últimas dos décadas en diversas escuelas de pensamiento ${ }^{6}$ que tienen como raíz común el uso del paradigma de la elección racional en contextos de competencia política. La escuela de la teoría espacial del voto (o la escuela clásica) emerge como el principal referente de la especialidad y corresponde además al modelo teórico sobre el cual desarrollamos nuestra aplicación empírica. A continuación se provee una versión simplificada de la teoría espacial del voto.

Existe un conjunto $I$ de votantes que tienen preferencias en un espacio $\Omega \subset \mathbb{R}^{d}$. Las preferencias son completas, transitivas y continuas, por lo tanto pueden ser representadas por funciones de utilidad. Existe un conjunto $K$ de candidatos que señalizan posiciones de política pública en el espacio $\Omega$.

La utilidad que el votante $i$ obtendría si el candidato $k$ fuera electo está dada por $u\left(\Pi_{k}, Z_{i}\right)=-\left\|\Pi_{k}-Z_{i}\right\|^{2}=-\left[\left(\pi_{k 1}-z_{k 1}\right)^{2}+\ldots+\left(\pi_{k d}-z_{i d}\right)^{2}\right]$.

\footnotetext{
${ }^{5}$ El distrito estudiado es el número veinte, correspondiente a las municipalidades de Maipú, Cerrillos y Estación Central.

${ }^{6}$ Las tres principales escuelas son la escuela del ciclo político económico (visión macro), la escuela de la teoría de juegos y señales aplicada a contextos políticos y la teoría clásica espacial del voto inicialmente desarrollada por Downs (1957).
} 
Donde $\Pi_{\mathrm{k}} \subset \mathbb{R}^{d}$ es la posición del candidato $k$ en el espacio $\Omega$ y $Z_{i}$ es la posición preferida por el votante $i$ en el espacio $\Omega^{7}$.

$\Pi_{\mathrm{k}}$ es una variable aleatoria que tiene una distribución de probabilidades de rango acotado que se mueve en función de la información pasada — si existe - respecto de las posiciones de política pública de un candidato y la señalización que el candidato entrega a sus electores a través de un proceso de actualización bayesiano. $\Pi_{\mathrm{k}}$ se determina después de un proceso de señalización de los candidatos. Esta situación requiere que usemos en nuestro análisis la utilidad esperada por el votante $E\left(u\left(\Pi_{\mathrm{k}}, Z_{i}\right)\right)$.

Por otro lado, el candidato $k$ recibe aportes monetarios de privados para señalizar su posición de políticas públicas en $\Omega$ por lo tanto tiene una restricción presupuestaria que le dice que no puede gastar más de lo que recibe de todos sus aportantes ${ }^{8} m_{k}$. Sea $s_{k}$ lo que el candidato $k$ gasta en señalizar su posición en $\Omega$ y sea $o_{k}^{q}$ lo que el candidato $k$ gasta en producir ruido a la señalización de su oponente $q$, entonces su restricción presupuestaria es $m_{k} \geq s_{k}+\sum_{(q \neq k) \in K} o_{k}^{q}$.

Definamos $\Theta$ como el diferencial neto de la utilidad esperada para el votante $i$ del candidato $k$ respecto de todos los otros candidatos, entonces

$$
\Theta\left(\Pi_{\mathrm{k}}\left(s_{k}, o_{k}^{q}\right), Z_{i}\right)=E\left(u\left(\Pi_{\mathrm{k}}\left(s_{k}, o_{k}^{q}\right), Z_{i}\right)\right)-\sum_{(q \neq k) \in K} E\left(u\left(\Pi_{\mathrm{q}}\left(s_{q}, o_{q}^{j}\right), Z_{i}\right)\right)
$$

Sea $F(\cdot)$ la función de densidad acumulada de la probabilidad de que el votante $i$ vote por el candidato $k$ dado el $\Theta\left(\Pi_{k}\left(s_{k}, o_{k}^{q}\right), Z_{i}\right)$. Entonces, en equilibrio de competencia política espacial tenemos que cada candidato $k$ maximiza $\sum_{i \in I} F\left(\Theta\left(\Pi_{\mathrm{k}}\left(s_{k}, o_{k}^{q}\right), Z_{i}\right)\right)$ sujeto a $m_{k} \geq s_{k}+\sum_{(q \neq k) \in K} o_{k}^{q}$. El equilibrio genera un vector con los $S^{*}$ y $O^{*}$ óptimos para todos y cada uno de los candidatos en competencia.

Si definimos como $n_{k}$ al número de votantes cuya distancia euclidiana desde sus posiciones ideales hasta la posición de señalización del candidato $k$ en el espacio $\Omega$ es mínima respecto de los demás candida-

\footnotetext{
${ }^{7}$ Claramente la utilidad es maximizada en cero, es decir, cuando el candidato $k$ señaliza exactamente la posición más preferida por el votante $i$.

${ }^{8}$ Para simplificar la exposición, los aportes son tratados como exógenos. Para ver un modelo de equilibrio general con aportes endógenos consultar Bonilla (2004).
} 
tos, entonces el candidato ganador es el que tiene asociado el mayor $n_{k}$ donde $\sum_{k \in K} n_{k}=\operatorname{Card}(I)$.

\section{DATOS Y METODOLOGÍA}

Los datos fueron obtenidos de una de las empresas líderes en investigaciones de mercado y de opinión pública en Chile que nos pidió reservar su nombre en el presente estudio, lo cual obviamente respetamos. En todo caso, la metodología de la encuesta responde a los más altos estándares profesionales en la materia. La encuesta arroja puntajes de termómetro en una escala de uno a siete para cada uno de los potenciales candidatos a la presidencia de la república.

La base de datos corresponde a una encuesta realizada en toda la Región Metropolitana ${ }^{9}$ durante el mes de enero del 2003. El tamaño de la muestra es de 1.011 datos, de los cuales se han eliminado los que respondieron en blanco alguno de los puntajes de termómetro, lo cual redujo el tamaño de la muestra a 793 datos válidos. El puntaje de termómetro se obtiene cuando el entrevistado responde a la siguiente pregunta:

"Pensando en el bienestar suyo y de su familia, ¿cómo evaluaría usted a las siguientes personalidades como Presidente de la República?”

La respuesta a la anterior pregunta provee el puntaje de termómetro que el votante individual asigna al candidato. Este puntaje, que va entre uno y siete, es utilizado en el análisis de factores para determinar las dimensiones latentes que explican las variaciones en el puntaje de termómetro. En nuestro caso los resultados revelan la existencia de dos dimensiones relevantes que explican más del $90 \%$ de las variaciones de la variable endógena, lo cual es absolutamente concordante con la literatura internacional que ocupa la metodología Cahoon-Hinich para mapear espacios electorales. Para una revisión más detallada de la metodología ver Apéndice al final del trabajo.

\section{RESULTADOS OBTENIDOS}

\subsection{Candidatos y contexto político de la muestra}

Mainwaring y Torcal (2003) estudian el impacto del legado político que el régimen autoritario dejó en la política chilena. Su trabajo se centra en

${ }^{9}$ La Región Metropolitana contiene el 40\% de la población del país, además los resultados de las elecciones presidenciales nacionales coinciden consistentemente con los de la Región Metropolitana. 
la reorganización y reestructuración de los partidos políticos en la nueva democracia instalada a partir de 1990. Uno de los hallazgos importantes es la constatación de la persistencia de la polarización observada en el espectro político chileno. Como sus resultados se basan en datos obtenidos en 1995, ocho años después podríamos presumir que, en la medida en que nos alejamos de la transición y nos acostumbramos a vivir en esta renovada democracia, el nivel de polarización debería probablemente reducirse en el tiempo.

Antes de pasar a los resultados obtenidos presentaremos a los potenciales candidatos a la presidencia de Chile evaluados en nuestra muestra de votantes. Reiteramos que la muestra fue tomada en enero del año 2003 y, por lo tanto, los candidatos evaluados son aquellos que aparecían como actores relevantes en ese momento.

En la Concertación, los candidatos que se incluyeron son los siguientes. La militante de la Democracia Cristiana (PDC, partido de centro e integrante de la Concertación) Soledad Alvear (S), quien a la fecha de la encuesta se desempeñaba con éxito como destacada ministra de Relaciones Exteriores del gobierno del Presidente Lagos, cosechando tratados y acuerdos internacionales de relevancia para el país. También en la Democracia Cristiana, se incluye en la muestra al ex presidente Eduardo Frei Ruiz-Tagle (F), quien había señalizado intenciones de volver a asumir liderazgos activos e incluso presidenciales. En el Partido Socialista (PS), entidad más a la izquierda con representación parlamentaria ${ }^{10}$, se incluyó a su destacado líder Camilo Escalona (E), quien ha sido en el pasado, y también con posterioridad a nuestra encuesta, presidente de dicho partido que forma parte de la coalición de gobierno. También en el sector de la Concertación se incluye una figura de perfil y liderazgo diferente, como Guido Girardi (G), actual senador por Santiago, líder del Partido por la Democracia (PPD), institución instrumental y progresista con un posicionamiento ciudadano que a esa fecha no había sido cuestionado por posibles problemas de probidad, como ha sucedido en los últimos años.

En la oposición, los candidatos incluidos son tres. En primer lugar tenemos a Andrés Allamand (A), ex presidente de Renovación Nacional (RN, partido de centro derecha), político joven y uno de los líderes de la derecha liberal chilena, quien a la fecha del estudio se encontraba parcialmente alejado de la política partidista y dedicado prioritariamente a la vida académica y profesional. En segundo término, el empresario y ex presidente de RN Sebastián Piñera (P), quien a esa fecha podía constituirse como una

${ }^{10}$ El Partido Comunista existe en Chile pero no tiene representación parlamentaria a la fecha de la muestra. 
opción presidencial. Por último, la muestra incluye a quien a esa fecha era el líder de la oposición y quien había sido el candidato presidencial el año 1999, cuando obtuvo el 47\% de los votos, Joaquín Lavín (L), militante de la Unión Demócrata Independiente (UDI).

Como explicamos anteriormente, a la fecha de la encuesta la actual Presidenta de la República, Michelle Bachelet Jeria, no obtenía todavía un lugar destacado en las encuestas. Ello explica el hecho de que no fuera incluida en nuestra medición. Es interesante analizar el surgimiento de Michelle Bachelet como candidata dentro de este contexto. Al momento del estudio, la señora Bachelet ejercía el cargo de Ministra de Defensa y comenzaba recién a destacar en los medios de comunicación. Al revisar los estudios de opinión pública en los meses y años que siguen, se aprecia un crecimiento explosivo de la opción presidencial de Michelle Bachelet ${ }^{11}$. El aporte de este trabajo consiste, en lo relativo a Michelle Bachelet, en explicar por qué se dieron las condiciones que permitieron el surgimiento de una mujer con su perfil como la mejor opción presidencial de la Concertación, incluso por sobre otra destacada mujer y ministra como Soledad Alvear, quien, según los resultados del presente estudio, a la fecha de la muestra representaba la mejor opción para la Concertación.

\subsection{Resultados}

Con este cuadro político en mente, describimos y analizamos los resultados de nuestro estudio. La implementación computacional de la metodología Cahoon-Hinich la realizamos a través del programa Map.exe desarrollado por Mel Hinich usando el lenguaje Fortran y que está disponible a través de los autores para ser aplicado por otros investigadores.

La Tabla $\mathrm{N}^{0} 1$ resume los resultados tras la aplicación de la metodología estadística ya mencionada a nuestros datos.

La literatura empírica del modelo espacial de votaciones (ver Enelow y Hinich, 1984 y 1994; Hinich, Khmelko y Ordershook, 1999) concuerda en que sólo dos dimensiones son suficientes para explicar más del 90\% de las variaciones de la variable endógena (puntaje de termómetro asignado por el votante a cada candidato) y que estas dos dimensiones son primeramente una dimensión en el eje político-económico clásico de izquierda-derecha, y una segunda dimensión que representa la posibilidad de reforma desde el

\footnotetext{
${ }^{11}$ Ver, por ejemplo, estudios de Opinión Pública del Centro de Estudios Públicos de junio-julio 2004 y siguientes para analizar dicha evolución.
} 
TABLA N ${ }^{\circ} 1$

\begin{tabular}{ccc}
\hline Candidato & $\begin{array}{c}\text { Posición en } \\
\text { dimensión 1 }\end{array}$ & $\begin{array}{c}\text { Posición en } \\
\text { dimensión 2 }\end{array}$ \\
\hline S & & \\
A & 0,58 & 5,99 \\
E & 4,00 & 3,04 \\
F & 1,14 & 2,06 \\
G & 0,61 & 2,81 \\
L & 0,93 & 2,49 \\
P & 4,55 & 5,49 \\
\hline
\end{tabular}

statu quo. Muchas veces esta segunda dimensión de reforma es vista como la percepción que tienen los votantes de la capacidad del candidato de terminar con los problemas de estancamiento y corrupción que cualquier gobierno enfrenta al término de su mandato. Debemos hacer notar que una de las limitaciones de nuestro trabajo es la carencia de información adicional en esta muestra particular que nos permita inferir endógenamente las dimensiones del mapa espacial. Sin embargo, dicha información no es necesaria si aceptamos los principales resultados de la literatura previa internacional y nos apoyamos en los resultados de las encuestas del CEP que a esa fecha ya mostraban una posición aventajada de Soledad Alvear y la irrupción de Michelle Bachelet.

La encuesta CEP entregada en enero del 2003 (realizada en diciembre del 2002) muestra una evaluación positiva de Soledad Alvear (73\%) y de Michelle Bachelet (72\%), muy por sobre cualquier otro potencial presidenciable de la Concertación en diciembre del 2005 (ningún otro supera el 50\%). Adicionalmente vemos que los temas relevantes para el electorado por esa fecha son de dos tipos principalmente. Los temas relacionados con el ámbito económico (empleo, pobreza, salud y vivienda) y los temas relacionados con capacidad del Estado de hacer bien las cosas (delincuencia y corrupción). Los primeros son temas fundamentales para definir el eje políticoeconómico izquierda-derecha, es decir la primera dimensión del mapa espacial. La segunda clase de temas guardan relación con la percepción de hacer bien las cosas, de reformar el statu quo. Si un candidato es percibido como un jugador diferente, a pesar de pertenecer a la coalición gobernante, ese jugador provee esperanzas de reformar lo que no ha resultado bien hasta ahora, de entregar un nuevo aire de cambio de combatir eficientemente la corrupción, etc. 
En primer término, nuestros datos nos permiten ubicar a los candidatos en el tradicional eje político-económico denominado izquierda-derecha.

Al observar los resultados obtenidos (Figura $\mathrm{N}^{0} 1$ ) apreciamos que en la primera dimensión el eje izquierda-derecha separa dos grupos de datos. Por un lado tenemos a los candidatos S, F, G, E agrupados a la izquierda. Estos candidatos pertenecen a la gobernante Concertación de Partidos por la Democracia. A la derecha de este grupo se ubican A, P y L, tres figuras de la derecha chilena que pertenecen a la Alianza por Chile.

FIGURA No 1

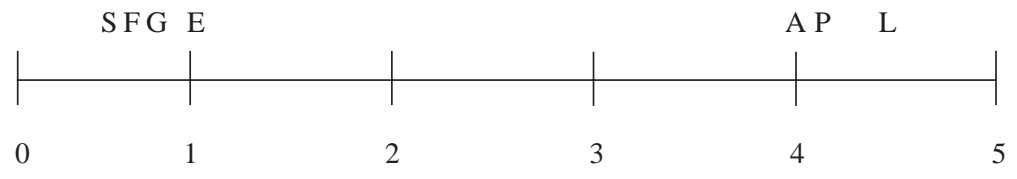

Nuestros resultados claramente apoyan la conclusión de Maiwaring and Torcal (2003), pues demuestran la existencia de un grado de polarización relativamente importante en la sociedad chilena, polarización que proviene de los años sesenta pero que alcanzó su máxima expresión durante el régimen militar instaurado en Chile entre 1973 y 1990 (ver González, 2000). Es importante mencionar que en el momento en que se tomó la encuesta no existía campaña política alguna, por lo tanto los resultados obtenidos reflejan el espectro político chileno sin ruido. Durante el período de campaña probablemente se dio algo parecido a lo que ocurrió el año 1999, cuando los dos candidatos con posibilidades reales de ganar la elección (Ricardo Lagos y Joaquín Lavín) adoptaron estrategias electorales moderadas con un discurso cercano al centro, por lo que podemos esperar que en la medida en que se acercan las elecciones, y dadas las estrategias de los candidatos que obviamente tratarán de convencer al decisivo votante mediano, los votantes perciban que los candidatos se asemejan entre sí en sus posiciones de políticas públicas, lo cual debiese verse reflejado en una menor polarización en las proximidades a una elección. Sin embargo, esta conjetura debe ser probada empíricamente en investigaciones futuras. Por ahora lo importante es la constatación de que los votantes perciben a los candidatos en dos grandes grupos. En un grupo están los de derecha, quienes fueron parte o apoyaron al régimen militar con sus políticas económicas neoliberales enfocadas en el crecimiento y con menor énfasis en los temas de distri- 
bución de la riqueza. En el otro grupo están los candidatos de la Concertación, quienes fueron opositores al régimen militar y promueven un concepto del crecimiento con igualdad, aunque ello implique potencialmente menor crecimiento. La existencia de estos dos grupos, claramente distantes y diferenciados para los votantes, apoya la tesis de polarización de Mainwaring y Torcal.

Como señalamos antes, las decisiones de votación no se toman en base a la consideración de una sola dimensión, sino que el $90 \%$ de las variaciones de la variable endógena puede explicarse en el análisis de dos dimensiones, siendo la segunda dimensión el eje reforma versus statu quo, según lo ha demostrado la literatura internacional de la teoría espacial del voto (ver Karkoglu y Hinich, 2006). La Figura No 2 presenta el posicionamiento de los mismos actores en la dimensión reforma.

FIGURA No 2

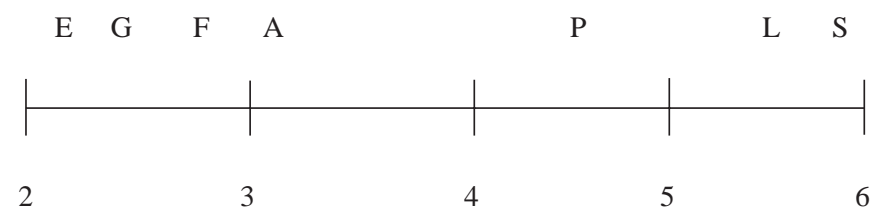

$\mathrm{Al}$ analizar la segunda dimensión (reforma versus statu quo), podemos apreciar claramente que los candidatos de derecha representan para los votantes un cambio importante. Éste era un resultado esperable, dado el tiempo que la coalición gobernante llevaba en el poder. Sin embargo Soledad Alvear, la ministra estrella del gabinete de la época, también es percibida como una candidata de cambio para los votantes. Nuestra interpretación de este hecho es que una candidata mujer, a pesar de ser ministra del gobierno de la época, representaba algo muy novedoso para la sociedad chilena, una sociedad muy tradicional y donde es muy raro encontrar a mujeres en altos puestos de poder en los ámbitos tanto público como privado.

Esta característica pudo ser un factor decisivo a la hora de decidir la nominación presidencial del candidato de la alianza gobernante, pues ayuda a contrarrestar uno de los argumentos fuertes de la oposición en la elección pasada: “el cambio que el país necesita”. 


\subsection{Un contexto favorable para el surgimiento de la candidatura de Michelle Bachelet}

Lamentablemente nuestra encuesta no consideró a otra mujer que entró con fuerza en al escenario político posterior. Se trata de la actual Presidenta de la República Michelle Bachelet, socialista, ex ministra de Salud y de Defensa y que en enero del 2003 aún no destacaba mucho en las preferencias del electorado.

Quizás uno de los resultados más interesantes que presenta este trabajo es ofrecer una explicación para el surgimiento exitoso de la candidatura de Michelle Bachelet a la presidencia.

Para ello integramos en un solo mapa espacial las dos dimensiones del análisis, esto es, el eje izquierda-derecha y el eje reforma versus statu quo (Figura $\mathrm{N}^{\circ}$ 3).

FIGURA N ${ }^{\circ} 3$

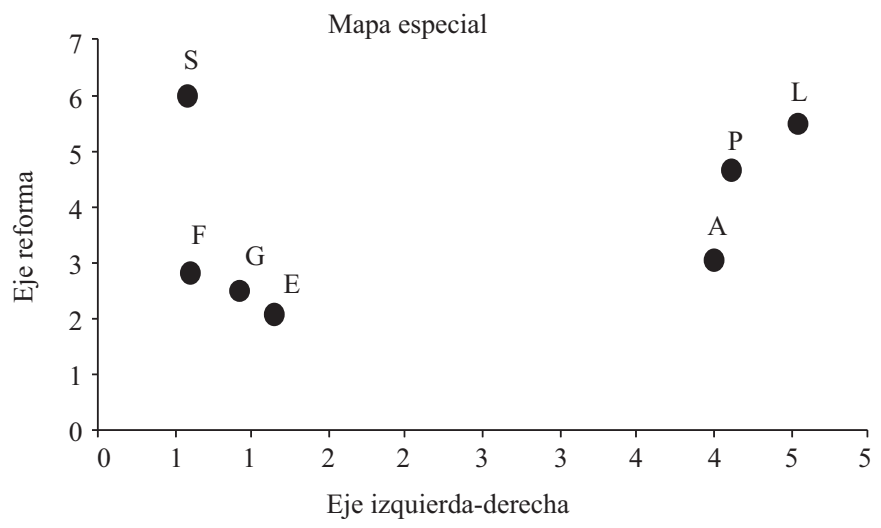

El análisis integrado de los ejes o dimensiones nos permite identificar de mejor forma la situación de los candidatos.

En efecto, una primera mirada muestra más posibilidades de éxito para Soledad Alvear en la Concertación y para Joaquín Lavín y Sebastián Piñera en la Alianza. Esto porque tienen posiciones similares a las de otros candidatos de su coalición en el eje izquierda-derecha, pero tienen una posición mucho más destacada en el eje reforma versus statu quo. 
Al contrastar estos resultados con la realidad histórica del momento, cabe recordar que los años 2003 y 2004 (hasta el día de las elecciones municipales) representan los años de mejor rendimiento de la Alianza en los estudios de opinión sobre elecciones presidenciales ${ }^{12}$.

La Concertación, por su parte, se encontraba en un momento complejo derivado de diversos factores, a saber: muchos años en el gobierno, problemas de corrupción de mayor y menor índole, problemas pendientes de solución y que inciden en las expectativas de los ciudadanos.

Este contexto político influyó en el perfil de los candidatos que tendrían potencial de ser exitosos en la elección presidencial de diciembre de 2005. Cabe recordar que en 1999 la Alianza logró capturar la dimensión reforma con el proyecto liderado por Joaquín Lavín. El año 2005 presentó un nuevo escenario en el cual la Alianza tenía menos fuerza en el eje reforma con la candidatura de Lavín (lo que puede explicar el avance de Piñera) y la Concertación tenía también dificultad para perfilar uno de sus candidatos tradicionales en la esfera presidencial.

El mapa político, en sus dimensiones izquierda-derecha y reforma versus statu quo, permite sugerir que los años 2003 y 2004 generaron un contexto apto para el surgimiento de la candidatura presidencial de Michelle Bachelet, quien logró, por sobre otros méritos, capturar con fuerza el eje reforma en la elección presidencial.

\section{CONCLUSIONES}

El presente trabajo ha tenido por objeto investigar si aún son válidas las conclusiones de Mainwaring y Torcal respecto a la polarización política existente en Chile como legado del régimen militar. Hemos aplicado la metodología de Cahoon-Hinich, ampliamente usada en economía política aplicada, sobre una base de datos de enero del 2003 de toda la Región Metropolitana y hemos construido un mapa espacial en dos dimensiones con quienes eran los potenciales candidatos a la presidencia de la república al 2003.

Nuestro principal resultado muestra que los votantes perciben a los candidatos de las dos coaliciones políticas muy distanciados y diferenciados en sus posiciones de política pública en la dimensión político-económica. Este distanciamiento y la diferenciación apoyan la tesis de Maiwaring y Torcal incluso para el año 2003, es decir que después de trece años del término del gobierno militar persisten elementos de polarización en la socie-

${ }^{12}$ Ver "Estudio Nacional de Opinión Pública”, diciembre del 2003 y junio-julio del 2004, Centro de Estudios Públicos. 
dad chilena que no han desaparecido a pesar del tiempo transcurrido. Nuestra conjetura es que a medida que se acercan las elecciones, las estrategias electorales de los candidatos deberían moverlos más al centro de la distribución y, una vez que ha pasado la elección, los candidatos deberían volver a moverse nuevamente hacia los extremos. Esta dinámica debiese empezar a decrecer a medida que las nuevas generaciones, que no vivieron la convulsión política de los años sesenta y que eran niños durante el régimen militar, comiencen a irrumpir con fuerza en las posiciones de poder de los partidos, y comiencen a perfilarse ellos como los futuros candidatos a los puestos políticos clave en los poderes del Estado. Algo que a nuestro juicio ha empezado tímidamente a suceder en los últimos años.

Por otro lado, al incorporar el eje reforma al análisis, se observa que los candidatos de la oposición son vistos, como era de esperar, como una alternativa de cambio para los electores. Sin embargo, Soledad Alvear, la única mujer incluida en la muestra, también es vista como una candidata capaz de proveer cambio para los electores, lo cual aporta un elemento importante a considerar por los partidos que componen la coalición de gobierno a la hora de elegir al candidato o la candidata presidencial del sector. Ello podría explicar el surgimiento exitoso de Michelle Bachelet como candidata presidencial de la Concertación.

\section{APÉNDICE}

La metodología Cahoon-Hinich se basa en el siguiente modelo estadístico:

Primero, sea $\Pi$ una matriz de orden $d x k$ que contiene las posiciones de los $k$ candidatos en las $d$ dimensiones de política pública de interés para los votantes. Las dimensiones son una forma genérica de denotar los temas de política pública de interés para los electores a la hora de decidir por quién marcar su voto. En la práctica $d=2$, donde la primera dimensión es siempre la percepción que los votantes tienen de la ubicación en el eje izquierda-derecha de cada candidato, y la segunda dimensión es la percepción de los votantes respecto a la capacidad que tiene el candidato de renovar sus equipos e ideas lejos de la desidia o malas prácticas asociadas a mantenerse en el poder por cierto tiempo, es decir, de la capacidad de proveer reformas (ver Lin et al., 1996, Hinich et al., 1999, o Karkoglu y Hinich, 2006).

Cada candidato tiene un vector $\Pi_{\mathrm{k}}$ de orden $d x 1$ que representa su respectiva posición en $\Omega$. Sea $Z$ una matriz de orden $d x n$ que contiene los 
puntos ideales de política pública en las $d$ dimensiones para los $n$ votantes de la muestra, cada votante tiene asociado su respectivo vector $Z_{i}$ de orden $d x 1$.

Segundo, sea $T$ una matriz de orden $k x n$ que contiene los puntajes de termómetro que cada votante en la muestra asigna a cada candidato (en particular $t_{k i}$ es el puntaje que el votante $i$ le asigna al candidato $k$ ).

El modelo empírico aplica una función de utilidad que es una distancia euclidiana entre la ubicación del candidato y la del votante

$$
t_{k i}=c_{k i}-\left[\left(\pi_{k 1}-z_{k 1}\right)^{2}+\ldots+\left(\pi_{k d}-z_{i d}\right)^{2}\right]=c_{k i}-\left\|\Pi_{k}-Z_{i}\right\|^{2}
$$

donde $c_{k i}$ es una variable aleatoria independiente de $t_{k i}, \pi_{k j}$ y $z_{i j}$ para $j=1,2, \ldots, d$. El error aleatorio $c_{k}$ tiene una varianza y $\psi_{k}^{2}$ que no está correlacionada entre los candidatos.

Expandiendo la ecuación (1) obtenemos

$$
t_{k i}=c_{k i}-\Pi_{k}^{\prime} \Pi_{k}+2 \Pi_{k}^{\prime} Z_{i}-Z_{i}^{\prime} Z_{i}
$$

Tercero, llamemos $\Sigma$ a la matriz de varianzas y covarianzas de $Z_{i}$ y suponemos que es diagonal cuyos elementos son $\sigma_{k}^{2}(j=1,2, \ldots, d)$. Además denotamos por $u_{j}$ al valor esperado ${ }^{1}$ de $z_{i j}(j=1,2, \ldots, d)$.

Cuarto, para convertir el modelo a uno lineal en $Z_{i}$, elegimos un candidato de referencia o numerario cuyo puntaje será restado del resto de los candidatos. Dado que la asignación del origen es arbitraria, el origen se establece en $\pi_{0}=0$ como punto inicial de referencia

$$
t_{k i}-t_{0 i}=c_{k i}-c_{0 i}-\Pi_{k}^{\prime} \Pi_{k}+2 \Pi_{k}^{\prime} Z_{i}
$$

Ahora calculamos la media muestral de cada candidato y computamos la diferencia entre las medias muestrales de cada candidato y el de referencia

$$
\overline{t_{k}}-\overline{t_{0}}=\overline{c_{k}}-\overline{c_{0}}-\Pi_{k}^{\prime} \Pi_{k}+2 \Pi_{k}^{\prime} \bar{Z}
$$

después restamos (4) de (3) para obtener

$y_{k i} \equiv\left(t_{k i}-t_{0 i}\right)-\left(\overline{t_{k}}-\overline{t_{0}}\right)=2 \Pi_{k}^{\prime}\left(Z_{i}-\bar{Z}\right)+\left(c_{k i}-\bar{c}_{\mathrm{k}}\right)-\left(c_{0 i}-\bar{c}_{0}\right)$

${ }^{1}$ El subíndice $i$ es eliminado, pues tomamos el valor esperado a través de los votantes que componen la muestra aleatoria. 
Definimos el vector $Y_{i}$ para todos los candidatos dado el votante $i$, operando la ecuación (5) obtenemos

$$
Y_{i} \equiv 2 \Pi^{\prime}\left(Z_{i}-\bar{Z}\right)+\left(-c_{0 i}+\overline{c_{0}}\right) \mathbf{1}+\left(C_{i}-\bar{C}\right)
$$

para el votante $i$, donde $\mathbf{1}$ es un vector de orden $k x 1$ que contiene sólo números uno, $C_{i}$ es un vector de orden $k x 1$ del componente aleatorio que acompaña a cada votante y $\bar{C}$ un vector de orden $k x 1$ que contiene los errores promedio de cada candidato dada la distribución de votante.

Después calculamos la matriz de varianzas y covarianzas muestral de $Y_{i}$. Esta matriz de orden $k x 1$ converge en probabilidad a la matriz de varianzas y covarianzas poblacional cuando $n \rightarrow \infty$

$$
\Sigma_{y}=4 \Pi^{\prime} \Sigma \Pi+\psi_{0}^{2} 11^{\prime}+\psi
$$

donde $\psi$ es una matriz diagonal de orden $k x k$ de las varianzas de los $c_{j}(j=1,2, \ldots, k)$.

Note que (7) puede ser expresada como

$$
\Lambda C \Lambda^{\prime}+\psi
$$

donde la raíz simétrica de $\Lambda C \Lambda^{\prime}$ es $\Lambda C^{1 / 2}$. Lo cual nos permite aplicar sobre $Y_{i}$ el método de análisis de factores para encontrar estimadores de máxima verosimilitud de $\Lambda C^{1 / 2} \Gamma$, donde $\Gamma$ es la matriz de rotación ortogonal por encontrar ${ }^{2}$.

Finalmente, debemos estimar las posiciones ideales de los votantes. Para hacerlo corremos los mínimos cuadrados ordinarios sobre

$$
t_{k i}-t_{0 i}=y_{k i}+2 \hat{\pi} k_{k 1} z_{i 1}+2 \hat{\pi}_{k 2 z_{i 2}}+\ldots . .+2 \hat{\pi}_{k d z_{i d}}+u_{k i}
$$

donde $u_{i k}=c_{k i}-c_{0 i}$, y los gorros denotan los estimadores obtenidos de $\hat{\Lambda}$ después de aplicar el método de análisis de factores. De esta manera obtenemos un estimador de las posiciones más preferidas de cada votante en las $d$ dimensiones $\left(z_{1 i}, z_{2 i}, \ldots . ., z_{d i}\right)$.

\footnotetext{
${ }^{2}$ Las técnicas de análisis de factores están bien explicadas en Morrison (1976).
} 


\section{REFERENCIAS}

Black, Duncan (1958): The Theory of Committees and Elections. Cambridge, England: Cambridge University Press.

Bonilla, Claudio (2002): “A Micro Application of the Spatial Theory of Voting”. En Revista de Ciencia Política, Vol. 22, N 2.

- (2004): "A Model of Political Competition in the Underlying Space of Ideology”. En Public Choice, 121.

Cahoon, Lawrence (1975): "Locating a Set of Points Using Range Information Only". Tesis de doctorado, Carnagie-Mellon University.

Cahoon, Lawrence, Melvin J. Hinich y Peter Odershook (1978): “A Statistical Multidimensional Scaling Method Based on the Spatial Theory of Voting”. En P.C. Wang (ed.), Graphical Analysis Representation of Multivariate Data. New York: Academic Press.

Cox, Gary (1990): "Multicandidate Spatial Competition”. En James Enelow y Melvin Hinich (editores), Advances in the Spatial Theory of Voting. Cambridge: Cambridge University Press.

Davis, Otto y Melvin Hinich (1966): “A Mathematical Model of Policy Formation en Democratic Societies”. En Joseph Bernd (ed.), Mathematical Applications in Political Science, II. Dallas: Southern Methodist University Press.

- (1968). "On the Power and Importance of the Mean Preferences in a Mathematical Model of Democratic Choice”. En Public Choice, 5.

Dow, Jay (1998): “A Spatial Analysis of Candidates Competition in Dual Member Districts: The 1989 Chilean Senatorial Election”. En Public Choice, 97.

Downs, Anthony (1957): An Economic Theory of Democracy. New York: Harper and Row.

Enelow, James y Melvin Hinich (1984): The Spatial Theory of Voting: An Introduction. New York: Cambridge University Press.

- (1994): “A Test of the Predicted Dimension Model in Spatial Voting Theory”. En Public Choice, 78: 155-170.

Ghobarah, Hazem (2000): “A Statistical Assessment of the Spatial Model of Ideology”. Tesis de doctorado, The University of Texas at Austin.

González, Jorge (2000): "The Political Instinct: The Role of Deception in Chilean Transition to Democracy”. Tesis de doctorado, The University of Texas at Austin.

Hinich, Melvin J., Valeri Khmelko y Peter Odershook (1999): “A Spatial Analysis of Ukraine’s 1998 Parliamentary Election”. En Post-Soviet Affairs, 15.

Hinich, Melvin y Michael Munger (1994): Ideology and the Theory of Political Choice. Ann Arbor: University of Michigan Press.

Hotelling, Harold (1929): “Stability in Competition”. En Economic Journal, 39: 41-57.

Karkoglu, Ali y Melvin Hinich (2006): “A Spatial Analysis of Turkish Party Preferences”. En Electoral Studies, 25: 369-392.

Lin, Tse-Min, Yun-Han Chu y Melvin J. Hinich (1996): “Conflict Displacement and Regime Transition in Taiwan: A Spatial Analysis”. En World Politics, 48.

Mainwaring, Scott y Mariano Torcal (2003): "The Political Recrafting of Social Bases of Party Competition: Chile, 1973-1995”. En The British Journal of Political Science, 33. 
Morrison, Donald (1976): Multivariate Statistical Methods. NY.: McGraw-Hill, segunda edición.

Pappi, Franz Urban y Gabrielle Eckstain (1998): “Voters' Party Preference in Multiparty Systems and their Coalitions and Spatial Implications: Germany After the Unification”. En Public Choice, 97.

Poole, Keith y Howard Rosenthal (1984): “U. S. Presidential election 1960-1980: A Spatial Analysis”. En American Journal of Political Science, 28.

(1991): "Patterns of Congressional Voting”. En American Journal of Political Science, 35.

Romer, Thomas y Howard Rosenthal (1978): “The Political Resource Allocation, Controlled Agendas, and the Status Quo”. En Public Choice, 33. 